\title{
Progress of Science from Microscopy to Microarrays (Part 1): Diagnosis of Parasitic Diseases
}

\section{Ayan Dey, Sarman Singh}

Division of Clinical Microbiology, Department of Laboratory Medicine, All India Institute of Medical Sciences, New Delhi-110 029, India

Address for correspondence: Prof. Sarman Singh, Email: sarman_singh@yahoo.com

\begin{abstract}
Even though description of the magnifying glass goes back to 1021 by an Arabic physicist in his book, Antony van Leeuwenhoek was the first man to improve the then simple microscope for viewing biological specimens in 1674. This suggests that every discovery has scope for improvement, be it physics or be it biology. In the field of biology, scientists have long studied gene expression as a hallmark of gene activities reflecting the current cell conditions and response to host immune defense systems. These studies have been cumbersome, technically demanding and timeconsuming. Application of microarrays has revolutionized this field and help understand the simultaneous expression of thousands of genes in a single sample put onto a single solid support. It is also now possible to compare gene expression in two different cell types, different stages of life cycle or two tissue samples, such as in healthy and diseased ones. Thus microarrays are beginning to dominate other conventional and molecular diagnostic technologies. The microarrays consist of solid supports onto which the nucleic acid sequences from thousands of different genes are immobilized, or attached at fixed locations. These solid supports themselves are usually glass slides, silicon chips or nylon membranes. The nucleic acids are spotted or synthesized directly onto the support. Application of microarrays is new for parasites. Most of these applications are done for monitoring parasite gene expression, to predict the functions of uncharacterized genes, probe the physiologic adaptations made under various environmental conditions, identify virulence-associated genes and test the effects of drug targets. The best examples are vector-borne parasites, such as Plasmodium, Trypanosoma and Leishmania, in which genes expressed, during mammalian and insect host stages, have been elucidated. Microarrays have also been successfully applied to understand the factors responsible to induce transformation from tachyzoite-to-bradyzoite and vice versa in Toxoplasma gondii. Thus microarrays provide a novel tool for diagnosis, prognosis and clinical management of infectious disease.
\end{abstract}

Keywords: Chips, expression, genes, parasites, reader, sequences, spotter

DOI: $10.4103 / 0974-2727.54800$

\section{INTRODUCTION}

C cientists have long studied gene expression as a hallmark of gene activities reflecting current cell conditions. However, this approach had been 'one-gene-one experiment'. By employing microarrays, researchers can decrease their reliance upon timeconsuming techniques; consequently, microarrays are beginning to dominate other molecular diagnostic technologies. Moreover, the ability of microarrays to monitor simultaneous gene expression of thousands of genes and to produce broad arrays of data has the potential to shift the resources of the scientists from data gathering to analysing data that are already available.
DNA microarrays are small, solid supports, onto which the sequences from thousands of different genes are immobilized, or attached, at fixed locations. These solid supports are usually glass slides, silicon chips or nylon membranes. The DNA is printed, spotted or synthesized directly onto these solid supports.

The design and construction of a DNA microarray for any given pathogen genome are used for monitoring the parasite gene expression. Also, one can predict the functions of uncharacterized genes, probe the physiologic adaptations made under various environmental conditions, identify virulenceassociated genes and test the effects of drugs. Similarly, by using host gene microarrays, one can explore host response at the level of gene expression and provide 
a molecular description of the events that follow infection. Host profiling might also identify gene expression signatures unique for each pathogen, thus providing a novel tool for diagnosis, prognosis and clinical management of infectious disease. ${ }^{[1]}$

\section{MICROARRAYS VIS-À-VIS CONVENTIONAL MOLECULAR METHODS}

Molecular methods, such as polymerase chain reaction (PCR), have aided in solving some of the sensitivity and specificity issues traditionally associated with the detection of parasites. A number of PCR-based assays, including gene amplification with specific primers, multiplex PCRs, restriction fragment length polymorphisms and real-time PCRs have been developed for the identification of parasite infections. However, the shortcomings of PCR-based assays become apparent during practical applications. The generation of nonspecific DNA fragments from environmental and clinical samples poses a significant problem often leading to false-positive results. Conversely, the failure to amplify a single diagnostic sequence due to inhibitors in the sample or possible mutations in the primer-binding region may result in false-negative results. Furthermore, although real-time PCR assays are sensitive enough to detect a single cell, the limited number of probes that can be applied in one reaction hinders its utility for confident multi-target detection and genotyping analyses.

The distinct advantage of microarray detection approach is that it combines powerful DNA amplification strategies with subsequent hybridization to oligonucleotide probes specific for multiple target sequences. This method allows for the simultaneous analysis of a larger number of genetic features in a single experiment. Thus, the amplification and hybridization approach produces a highly sensitive and specific platform with high-throughput capacity for pathogen detection and genotyping. Due to the increasing reliance upon genetic tests for identification and differentiation, the low concentration or number of organisms required in causing the disease, and the often-found presence of multiple parasites in a single environmental or clinical sample, such methods would be ideally suited for the detection of parasites. ${ }^{[2]}$

\section{APPLICATION OF MICROARRAYS IN THE PARASITIC DISEASES}

Traditional molecular methods work on a 'one gene - one experiment' basis and because of the limited throughput of such methods, the whole picture of gene function is difficult to obtain. Scientists have long studied gene expression as a hallmark of gene activities reflecting current cell conditions and response to host immune defense systems. These studies are cumbersome, technically demanding and timeconsuming. Application of microarrays is new for parasites. Most of these applications are done to monitor the parasite gene expression, predict the functions of uncharacterized genes, probe the physiologic adaptations made under various environmental conditions, identify virulenceassociated genes and to test the effects of drug targets. Similarly, by using host-specific gene microarrays, one can explore host response at the level of gene expression and provide a molecular description of the events that follow the parasitic infection. Host-specific gene profiling might also identify gene expression signatures unique for each pathogen or for specific life stage of the parasite. The best examples are vector-borne parasites like Plasmodium, Trypanosoma and Leishmania, in which genes expressed, during mammalian and insect host stages, have been elucidated. ${ }^{[3-5]}$ Microarrays have also been successfully applied to understand the factors responsible to induce transformation from tachyzoite-to-bradyzoite and vice versa in Toxoplasma gondii. ${ }^{[6]}$ In malaria and Trypanosoma cruri, parasitic genes have been identified, which are expressed during insect phase and mammalian host stages. ${ }^{[7]}$

\section{Malaria}

Initial studies using microarray was carried out on Plasmodium new gene discovery, and subsequently studies on patterns of coordinate gene regulation began. For instance, in a microarray of known Plasmodium falciparum cDNAs used to assess gene expression at five different stages of erythrocyte development, cluster analysis revealed common patterns of expression of groups of genes involved in carbohydrate metabolism, adhesion/invasion and translation machinery. This analysis demonstrated how multiple components of a pathway are expressed at the appropriate stage in a way not possible by earlier techniques that analysed gene expression one by one. As further advancement, a more sophisticated Plasmodium microarray spotted with open-reading frame (ORF)specific oligonucleotides showed coordinated expression of ribosomal proteins, tRNA synthetases, initiation and elongation factors, helicases and chaperones that expands the understanding of protein translation activity in the trophozoite stage. ${ }^{[3,5]}$

In Plasmodium falciparum, through differential hybridization and sequencing of relevant clones, large differences in gene expression were identified between the blood stage 
trophozoite form and the sexual stage gametocyte form. ${ }^{[8]}$ In case of Plasmodium, expression of around 5159 genes during the nine stages of parasite have been identified, and genomic microarray based on this information have been developed for diagnosing and studying drug resistance mechanisms. The utility of microarrays is not restricted to fully sequenced organisms. A powerful screening tool has been developed for Plasmodium falciparum. A DNA microarray of 3648 random genomic clones was used to identify more than 50 genes for which expression differed significantly between the trophozoite and gametocyte stages [Table 1].

\section{Toxoplasma}

The application of microarray analysis to T. gondii gene expression has also moved beyond validation and gene discovery alone. Changes in gene expression between the rapidly dividing tachyzoite stage and the slow-growing, immune-system-avoiding bradyzoites were evaluated on a cDNA array ${ }^{[6]}$ Cluster analysis of these results identified coordinate bradyzoite expression of clones, which encode cell-surface proteins that could aid in immune avoidance. Clustering also showed a group of bradyzoitedownregulated clones encoding metabolic enzymes that reinforces arguments for differences in sugar metabolism in this stage. Transiently expressed clones also cluster, which may be essential to the developmental process [Table 1]. The same microarray, used to compare global gene expression in wild-type cell lines with chemically induced, tachyzoite-to-bradyzoite differentiation-defective cell lines allowed the construction of a model of hierarchical gene activation during bradyzoite development. These results set the stage to focus on genes that occupy key positions in the developmental pathway of Toxoplasma. ${ }^{[6,9]}$

\section{Trypanosoma}

Amongst the other parasites, Trypanosoma cruz $i$ is the most recent protozoan parasite to be examined by microarray analysis. The study reported from six hybridizations using independently generated $T$. cruzi parasite samples consistently identified 60 probes that detected genes upregulated within $2 \mathrm{~h}$ after extracellular trypomastigotes were induced, in vitro, to differentiate into amastigotes. Sequence analysis from these 60 probes identified 14 known and 25 novel T. cruzi genes involved in this transformation. ${ }^{[10]}$ Similarly, a microarray of T. brucei genomic fragments has identified new genes whose expression differs between the insect-borne stage and the human-infectious stage of the parasite. Hybridizations identified $75 \%$ of the clones as expressed sequence, with 300 clones differentially expressed. The microarray results were verified by the expression pattern of known genes and northern blots or semi-quantitative RT-PCR for new clones. The results established the effectiveness of the microarray as a method to assess expression of a large collection of potential genes and the 20 new; differentially regulated genes described represents a substantial advance in the knowledge of T. brucei genomics. ${ }^{[7]}$

\section{Leishmania}

In the genus Leishmania, several studies have used microarray analysis. Using spotted arrays, changes in expression level in different stages of the parasite life cycles have been demonstrated. In Leishmania major, a randomly sheared genomic microarray was applied to the changes in gene expression in cultured parasites differentiating from the rapidly growing, procyclic stage to the infectious, metacyclic stage, both of which are found in the insect vector in nature. ${ }^{[1]}$ The microarray results showed upregulation in procyclic stage of genes that are known to be active in that stage, such as those encoding $\beta$-tubulin, histones and ribosomal proteins, supporting the validity of the technique. In metacyclic stage, HASP/geneB, SHERP/ geneD, META1 and HSP70 were among those found to

\begin{tabular}{|c|c|c|c|c|}
\hline Species & Microarray type & Array clones & Results & Genome sequenced \\
\hline Plasmodium falciparum & Genomic & 3648 & New genes & $100 \%$ \\
\hline Plasmodium falciparum & cDNA & 944 & New genes & $100 \%$ \\
\hline Plasmodium falciparum & Oligo & 6272 & Coordinate regulation & $100 \%$ \\
\hline Toxoplasma gondii & CDNA & 4319 & New genes, coordinate regulation & $100 \%$ \\
\hline Trypanosoma brucei & Genomic & 21024 & New genes & $100 \%$ \\
\hline Trypanosoma cruzi & Genomic and CDNA & 4321 & New genes & $\begin{array}{l}759.07 \text { bp sequences are covered using } \\
\text { whole genome shotgun approach }\end{array}$ \\
\hline Leishmania major & Genomic & 10464 & New genes & Nearly completed \\
\hline Leishmania major & cDNA & 2091 & New genes & Nearly complete \\
\hline Leishmania major & Genomic & 10479 & New genes & Nearly complete \\
\hline Leishmania donovani & Genomic & 2304 & New genes & Nearly complete \\
\hline
\end{tabular}


be upregulated. Many more differentially regulated clones were identified that did not have homology to proteins of known function. The validity of the microarray results was further affirmed by comparison with northern blots, with $33 / 47$ of the clones tested showing regulation as predicted. ${ }^{[4]}$ Thus, the technique was established as an effective tool to find new genes that are expressed in a pattern that suggests stage-specific importance. However, no pattern of coordinate expression of functionally related or pathway-specific genes was described.

A second application of the same randomly sheared genomic clones of L. major was also used to evaluate gene expression as procyclic promastigotes differentiated in culture into metacyclic promastigotes. ${ }^{[1]]}$ Though extensive homology searches using clones with statistically significant expression patterns assigned putative genes to functional categories, the major contribution of this study is in validation of the array and new gene discovery, more than in coordinate regulation. By applying complementary DNA (cDNA) and genomic microarrays for L. major, a number of new genes that are expressed in a stage-specific fashion have been identified, and preliminary results from an L. donovani genomic microarray has also demonstrated new gene discovery. Even though not much work has been done on leishmaniasis, in the next few years, building on this foundational work, microarrays are expected to solve several questions such as mechanism of drug resistance, why only $2-5 \%$ of VL cases develop post kala-azar dermal leishmaniasis?, which genes control differentiation from promastigote-to-amastigote stages and vice versa? And why some species cause visceral disease and others cause cutaneous and mucocutaneous form of disease? ${ }^{[12]}$

\section{Intestinal parasites}

Apart from vector-borne parasites, microarrays found application for the parallel detection and genotyping of intestinal parasite Entamoeba histolytica, Giardia lamblia, and Cryptosporidium parvum have also been developed. Recently, oligonucleotide microarrays have been used successfully for the detection of Entamoeba. ${ }^{[13]}$ Microarray-based genotyping assay can readily be applied to the study of E. histolytica clinical isolates to determine genetic diversity and potential genotypic-phenotypic associations. This technique combines powerful DNA amplification strategies with subsequent hybridization to oligonucleotide probes specific for multiple target sequences. The distinct advantage of this detection approach is that it can study thousands of genes simultaneously. In addition to conserved genes, such as rRNA and hsp, which have been widely used as diagnostic markers, many genus- and species- specific genes like intergenic region between the superoxide dismutase (sod) and actin genes, and the cysteine protease gene (cp1) were selected as amplification targets so as to avoid potential co-amplification and cross-hybridization issues. The amplification and hybridization of multiple diagnostic regions to short genus-, species- and subtypespecific probes allowed for the unequivocal detection and discrimination of E. histolytica, Entamoeba dispar, G. lamblia assemblages A and B, and C. parvum genotypes 1 and 2 in a single assay. Thus, this method may aid in confidently expediting the detection of these three major waterborne parasites while simultaneously providing valuable epidemiological information. ${ }^{[13]}$

Apart from Entamoeba, microarray found its application in the diagnosis of pathogenic protozoan parasite Microsporidium. By integrating extraction-free, FTA filter-based template preparation and amplification of homologous SSU rRNA sequences with universal primers, this microarray analysis strategy allowed to generate unique profiles so as to rapidly detect and differentiate four major species of microsporidia with greater sensitivity and specificity and high-throughput capability. ${ }^{[14]}$

PCR-based methods, such as conventional PCR, restriction fragment length polymorphism and real-time PCR, have greatly improved sensitivity and specificity to detect and differentiate microsporidian species in clinical and environmental samples. ${ }^{[15,16]}$ However, the PCR method usually requires setting up multiple reactions with different specific primer pairs so as to discern a specific species. But process is more cumbersome when a large amount of unknown samples are to be tested. However, in microarrays oligonucleotides amplification strategy is combined with subsequent hybridization to multiple specific probes. These probes are immobilized on the microchip as a means to simultaneously detect multiple species of microsporidia. The FTA filter-based template preparation strategy not only eliminates labor-intensive steps, such as sonication, freeze-thawing and glass bead disruption, used to extract DNA from microsporidian spores but also prevents significant DNA loss due to smaller sample sizes and effectively removes inhibitors from fecal samples. ${ }^{[17]}$

Compared with the commercial stool DNA extraction kit, this method has a lower cost, requires less technical training and equipment and can handle a larger number of samples at one time. ${ }^{[18]}$ By taking advantage of conserved sequences of SSU rRNA genes shared by all microsporidian species, it will be able to amplify 1300bp fragments from microsporidium-containing samples, followed by a second reaction using one degenerate 
primer to synthesize and fluorescently label single-stranded DNA from every primary PCR product. These products were then hybridized to specific oligonucleotide probes on the microarray. DNA-DNA hybridization limited nonspecific PCR fragments and yielded reliable sensitivity limits to a level of 10 Enterocytozoon intestinalis spores. Using the microarray as designed, identification of each microsporidian species was dependent not on a single amplicon but on a defined composite hybridization profile. Since each species has its own unique array pattern, it can be easily distinguished from another.

\section{CONCLUSION}

The relatively rapid and accurate nature of microarray platform has great potential in parasitic disease diagnosis and it holds great promise for progress in alleviating the suffering that results from infections with these parasites.

\section{REFERENCES}

1. Slonim DK. From patterns to pathways: gene expression data analysis comes of age. Nat Genet 2002;32:502-8.

2. Chizhikov V, Rasooly A, Chumakov K, Levy DD. Microarray analysis of microbial virulence factors. Appl Environ Microbiol 2001;67:3258-63.

3. Ben Mamoun C, Gluzman IY, Hott C, MacMillan SK, Amarakone AS, Anderson DL, et al. Co-ordinated programme of gene expression during asexual intraerythrocytic development of the human malaria parasite Plasmodium falciparum revealed by microarray analysis. Mol Microbiol 2001;39:26-36

4. Beverley SM, Akopyants NS, Goyard S, Matlib RS, Gordon JL, Brownstein $\mathrm{BH}$, et al. Putting the Leishmania genome to work: functional genomics by transposon trapping and expression profiling. Philos Trans R Soc Lond B Biol Sci 2002;357:47-53.

5. Bozdech Z, Zhu J, Joachimiak MP, Cohen FE, Pulliam B, DeRisi JL. Expression profiling of the schizont and trophozoite stages of Plasmodium falciparum with a long-oligonucleotide microarray. Genome Biol 2003;4:R9.
6. Cleary MD, Singh U, Blader IJ, Brewer JL, Boothroyd JC. Toxoplasma gondii asexual development: identification of developmentally regulated genes and distinct patterns of gene expression. Eukaryot Cell 2002;1:329-40.

7. Diehl S, Diehl F, El-Sayed NM, Clayton C, Hoheisel JD. Analysis of stage-specific gene expression in the bloodstream and the procyclic form of Trypanosoma brucei using a genomic DNA-microarray. Mol Biochem Parasitol 2002;123:115-23.

8. Hayward RE, DeRisi JL, Alfadhli S, Kaslow DC, Brown PO, Rathod PK. Shotgun DNA microarrays and stage-specific gene expression in Plasmodium falciparum malaria. Mole Microbiol 2000;35:6-14.

9. Singh U, Brewer JL, Boothroyd JC. Genetic analysis of tachyzoite to bradyzoite differentiation mutants in Toxoplasma gondii reveals a hierarchy of gene induction. Mol Microbiol 2002;44:721-33.

10. Minning TA, Bua J, Garcia GA, McGraw RA, Tarleton RL. Microarray profiling of gene expression during trypomastigote to amastigote transition in Trypanosoma cruzi. Mol Biochem Parasitol 2003;131:55-64.

11. Saxena A, Worthey EA, Yan S, Leland A, Stuart KD, Myler PJ. Evaluation of differential gene expression in Leishmania major Friedlin procyclics and metacyclics using DNA microarray analysis. Mol Biochem Parasitol 2003;129:103-14.

12. Singh S., Dey A, Sivakumar R. Applications of Molecular Methods for Leishmania control. Expert Rev Mol Diagn 2005;5:251-65.

13. Wang Z, Vora GJ, Stenger DA. Detection and Genotyping of Entamoeba bistolytica, Entamoeba dispar, Giardia lamblia, and Cryptosporidium parvum by Oligonucleotide Microarray. J Clin Mirobiol 2004;42:3262-71.

14. Wang Z, Orlandi PA, Stenger DA. Simultaneous detection of four human pathogenic Microsporidian Species from clinical samples by oligonucleotide microarray. J Clin Microbiol 2005;43:4121-8.

15. Franzen C, Muller A. Molecular techniques for detection, species differentiation, and phylogenetic analysis of microsporidia. Clin Microbiol Rev 1999;12:243-85.

16. Garcia LS. Laboratory identification of the microsporidia. J Clin Microbiol 2002;40:1892-901.

17. Orlandi PA, Lampel KA. Extraction-free, filter-based template preparation for rapid and sensitive PCR detection of pathogenic parasitic protozoa. J Clin Microbiol 2000;38:2271-7.

18. Subrungruang I, Mungthin M, Chavalitshewinkoon-Petmitr P, Rangsin R, Naaglor T, Leelayoova S. Evaluation of DNA extraction and PCR methods for detection of Enterocytozoon bienuesi in stool specimens. J Clin Microbiol 2004;42:3490-4.

19. Duncan R. DNA microarray analysis of protozoan parasite gene expression: outcomes correlate with mechanisms of regulation. Trends Parasitol 2004;20:211-6.

Source of Support: Nil, Conflict of Interest: None declared. 